\title{
Accuracy in diagnosis of postpartum haemorrhage using visual estimation of blood loss versus change in haematocrit in a tertiary teaching hospital in Tanzania
}

\author{
PETER J.T. WANGWE* and BELINDA BALANDYA
}

Department of Obstetrics and Gynaecology, School of Medicine, Muhimbili University of Health and Allied Sciences, P.O. Box 65117, Dar es Salaam, Tanzania

\begin{abstract}
Postpartum haemorrhage is the leading cause of maternal death in the developing country and yet is poorly diagnosed due to inaccurate measurement of blood loss following delivery. A study was carried out at Muhimbili National Hospital (MNH) Tanzania between October 2005 and January 2006 to determine the accuracy of visual estimation of blood loss (VEBL) in comparison to laboratory measurement of blood loss in diagnosis of primary postpartum haemorrhage (PPH). A total of 426 pregnant women who were in active phase of labour were recruited and their venous blood was drawn for estimation of haematocrit before delivery and 12 hours thereafter. Active management of third stage of labour was conducted by giving 10IU of oxytocin (intramuscularly or intravenously) and this was followed by visual estimation of blood loss. The proportion of patients who developed PPH was then determined by both methods. The mean duration of third stage of labour was 8.3 minutes and mean blood loss was $164.9 \mathrm{ml}$. The prevalence of PPH was $8.9 \%$ and $16.2 \%$ by VEBL and changes in haematocrit, respectively. Change in haematocrit in diagnosis of PPH was found to be more accurate, specific with high positive predictive values compared to VEBL. The need for additional uterotonics was $5.8 \%$ and the commonest labour complications associated with PPH were second degree tear, retained placenta and EUA for continued bleeding. In conclusion, VEBL using calibrated vessel will increase accuracy where conventional method using non calibrated method is used for diagnosis of PPH. Service providers working in labour wards need to be trained on how to estimate blood loss using simulated methods so as to increase their long term memory and accuracy in diagnosis of post-partum haemorrhage, hence provision of immediate intervention.
\end{abstract}

Keywords: visual estimation, blood loss, postpartum, haemorrhage, haematocrit, Tanzania

\section{Introduction}

Diagnosis of postpartum haemorrhage $(\mathrm{PPH})$ in poor resource countries where there is high prevalence of anaemia in pregnancy is a challenging problem especially when there are no symptoms of cardiac failure due to physiological compensatory mechanism which occurs immediately after delivery. PPH accounts for a quarter of all maternal deaths worldwide (Chong \& Su, 2006) but this tends to be underestimated in the poor resource countries. The high prevalence of anaemia among pregnant women in low income countries makes even a modest $\mathrm{PPH}$ more serious and life threatening. Mortality from PPH has remained high in low income countries despite international efforts to decrease maternal mortality since the launch of the Safe Motherhood Initiative in 1987 (Hofmeyr et al., 2009. At the Muhimbili National Hospital in Tanzania PPH is among the leading cause of maternal mortality and has been reported to be the second leading cause of emergency peripartum hysterectomy (Pembe et al., 2012). Active

"Correspondence: Peter Wangwe; Email. drwangwe@yahoo.com 
management of the third stage of labour (AMTSL) is the primary intervention known to reduce the incidence of PPH and the World Health Organization (WHO) and International Council for Midwifery (ICM) recommends this to be implemented using oxytocin (Anderson \& Etches, 2007). However discrepancy in diagnosis of PPH due to inaccurate in estimating actual blood loss (associated with observed error) where different methods has been employed limits accuracy in diagnosis of PPH hence ending up with postpartum complication (Rath, 2011). More importantly the greatest risk of maternal death is among women who deliver at home, either alone or assisted with traditional birth attendant (Prata et al., 2005).

In view of the magnitude of maternal mortality in Tanzania where majority of the patients are anaemic, there is a need to conduct regular on job training on methods of diagnosis of primary PPH and therefore increase accuracy in its diagnosis and provide early intervention. The objective of this study therefore was to determine the accuracy of diagnosis of PPH using Visual Estimation of Blood Loss (VEBL) in comparison with actual measurement of blood loss using laboratory investigation by determining drop in haematocrit twelve hours after vaginal delivery.

\section{Materials and Methods}

\section{Study Setting}

A prospective analytical study was conducted at the labour ward of the Muhimbili National Hospital (MNH) in Dar es Salaam, Tanzania. Muhimbili National Hospital is the largest referral hospital and a teaching hospital for the Muhimbili University of Health and Allied Sciences. About 20-30 deliveries take place in 24 hours with an average of 9,000-11,000 deliveries per year. The gold standard for estimation of blood loss after delivery in the hospital is through visual estimation (VEBL). There is no actual measurement of blood loss using the calibrated jar due to the difficulties encountered either blood mixed with liquor, urine and lack of enough staff working in this busy labour ward.

\section{Study population and data collection}

The study consisted of all pregnant women admitted in the active phase of labour between October 2005 and January 2006. During the study period there were 2882 delivery. However, 2487 women were excluded from the study due to not meeting the inclusion criteria. The exclusion criteria were women were women admitted in second stage of labour, at night, had preeclampsia, eclampsia and multiple pregnancies. A total of 426 women who were admitted in the hospital for delivery between 08.00 and 15.00 hours were recruited in the study. Their venous blood was drawn for estimation of haematocrit before delivery and 12 hours thereafter. A change of haematocrit of more than $10 \%$ after delivery was considered to be significant hence diagnosis of primary $\mathrm{PPH}$.

Active management of the third stage of labour was done by intramuscular (IM) administration of $10 \mathrm{U}$ of oxytocin one minute after delivery of the baby according to the hospital guidelines. The placenta delivered by controlled cord traction and that needed for additional uterotonic agents were assessed by manual palpation of the uterus NOT CLEAR. Estimation of blood loss was done visually as explained above. Any patient with VEBL more 
than 500ml was diagnosed to have primary $\mathrm{PPH}$ and if had poorly contracted uterus with continued bleeding was given additional uterotonics drugs after ruling out other causes of bleeding.

\section{Data analysis}

Of the recruited subjects, 12 women delivered by emergency caesarean section. In 11 women, haematocrit could not be traced 12 hours after delivery and eight women had some of their information missing hence these were excluded from the analysis. All data were entered into a computer using Epi-Info-6 software. Socio-demographic information including gestational age, parity, admitting haematocrit, visually estimated blood loss at delivery and the postpartum haematocrit 12 hours post-delivery were recorded. Haematocrit change was calculated by subtracting the 12 hours post-delivery haematocrit from the admitting haematocrit. The drop in haematocrit was divided to the admitting haematocrit then computed into percentage. The change of haematocrit of more than $10 \%$ was considered to be PPH. Data analysis was done using SPSS (Statistical Package13 for Social Sciences) programme. A p-value of less than 0.05 was considered statistically significant.

\section{Ethical consideration}

Ethical approval was provided by the Research and Publication Committee of the Muhimbili University of Health and Allied Sciences, Tanzania. All women gave informed consent after being informed in detail the aims of the study.

\section{Results}

Data from 395 women were analyzed and the average visual estimation of blood loss (VEBL) was $164.9 \pm 120.2 \mathrm{ml}$ with primary $\mathrm{PPH}$ rate of $8.9 \%$. The average 12 hours postpartum haematocrit change was 5.3 (range $=-0.48$ to 19.7 ) with primary PPH rate of $16.2 \%$ (Table1). The mean duration of third stage of labour was 8.3 minutes and the need for additional uterotonics was $5.8 \%$. The commonest labour complications encountered were second degree tear, retained placenta and EUA for continued bleeding; these are some of the causes of primary PPH.

Table 1: Characteristics of study participants and diagnosis of PPH by visual estimation of blood loss (VEBL) versus change in haematocrit

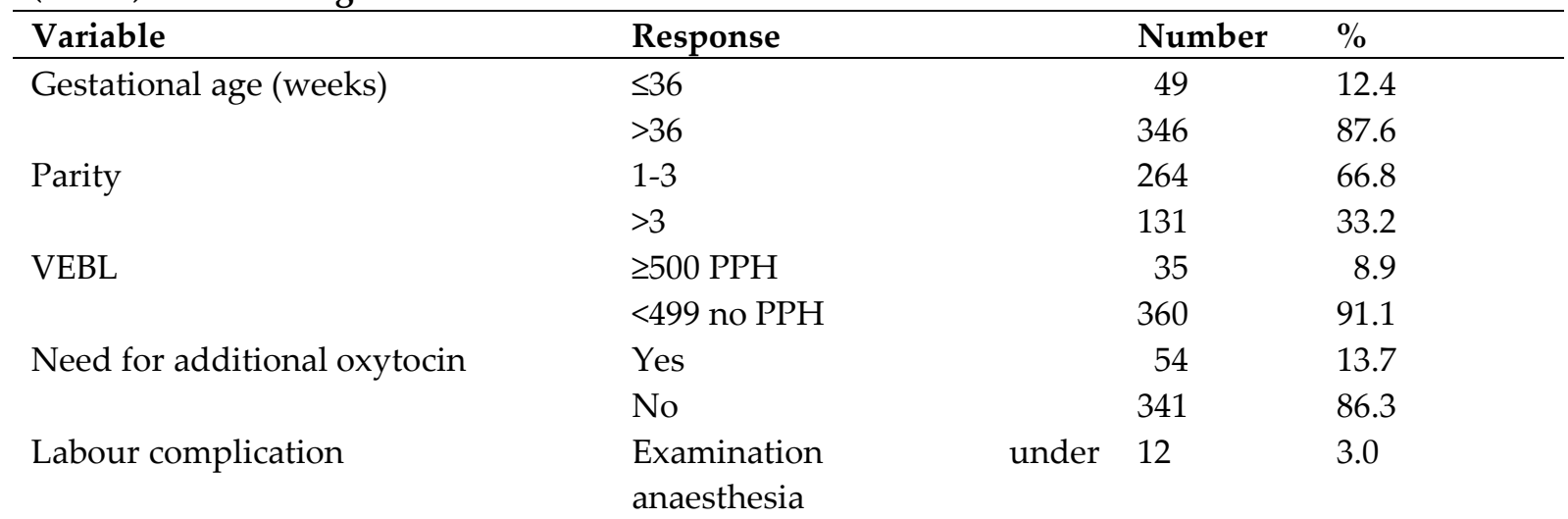




\begin{tabular}{|c|c|c|c|}
\hline & Retained Placenta & 11 & 2.8 \\
\hline & Cervical tear & 3 & 0.8 \\
\hline & Second degree tear & 14 & 3.6 \\
\hline \multirow[t]{2}{*}{ Duration of $3^{\text {rd }}$ stage of labour } & Normal (<15 min) & 343 & 86.8 \\
\hline & Prolonged ( $\geq 15 \mathrm{~min})$ & 52 & 13.2 \\
\hline \multirow[t]{2}{*}{ Mother on augmentation } & Yes & 100 & 25.3 \\
\hline & No & 295 & 74.7 \\
\hline \multirow[t]{2}{*}{ PROM } & Yes & 57 & 14.4 \\
\hline & No & 338 & 85.6 \\
\hline Mean blood loss (ml) & 164.9 & & \\
\hline Mean change in haematocrit & 5.3 & & \\
\hline
\end{tabular}

The proportion of women who had PPH as a result of change in haematocrit of more than $10 \%$ was higher compared to the routine diagnosis of primary PPH using VEBL (Tables 1 and 2). However the need for blood transfusion was very low $(1.9 \%)$. The minimum blood loss was reported to be $50 \mathrm{ml}$ whereas the maximum was $1120 \mathrm{ml}$. The mean haematocrit before and after delivery was 33.2 and 30.4, respectively. Less than half of the study population had less than 28 haematocrit before delivery with almost normal distribution curve. However, 12 hours after delivery half of the study population had less than 28 haematocrit with the distribution curve skewing to the right indicating significant change after delivery (Figures 1 and 2). The change in haematocrit was more accurate, sensitive with high positive predictive value in diagnosis of PPH compared to VEBL (Table 2).

Table 2: Haematocrit change and visual estimated blood loss (VEBL) in diagnosis of PPH

\begin{tabular}{llllllll}
\hline & PPH & No PPH & Sensitivity & Specificity & PPV & NPV & Accuracy \\
\hline VEBL & 35 & 360 & 34.3 & 47.7 & 8.86 & 83.04 & 45.9 \\
$\begin{array}{l}\text { Change in } \\
\text { haematocrit }\end{array}$ & & 328 & 65.7 & 52.3 & 16.9 & 91.1 & 54.1 \\
\hline
\end{tabular}

Key: $\mathrm{PPV}=$ positive predictive value; NPV= negative predictive value

When regression analysis for VEBL and change in haematocrit was used, there was a statistically significant correlation between the change in haematocrit and the VEBL (Pearson's correlation $\mathrm{r}=0.286, P<0.01)$. The T-statistic value for VEBL was the smallest at -5.324 negative and very significant. This indicated that all patients who had PPH by VEBL $(8.9 \%)$ had a drop in haematocrit. There was a steady decline in the haematocrit change with an increase in the average VEBL. 


\section{estriated blood loss}

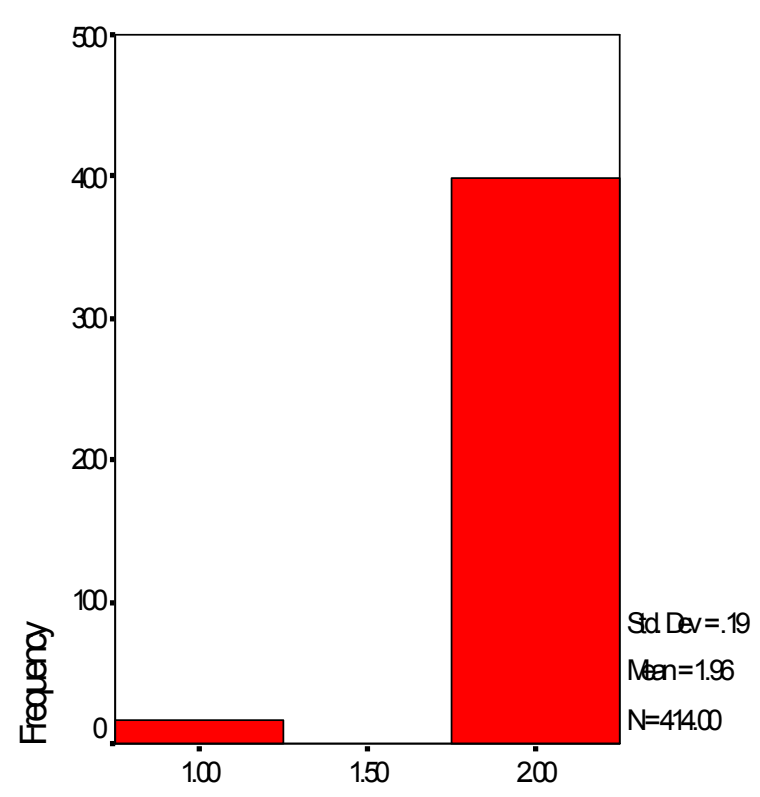

estrated bloodloss

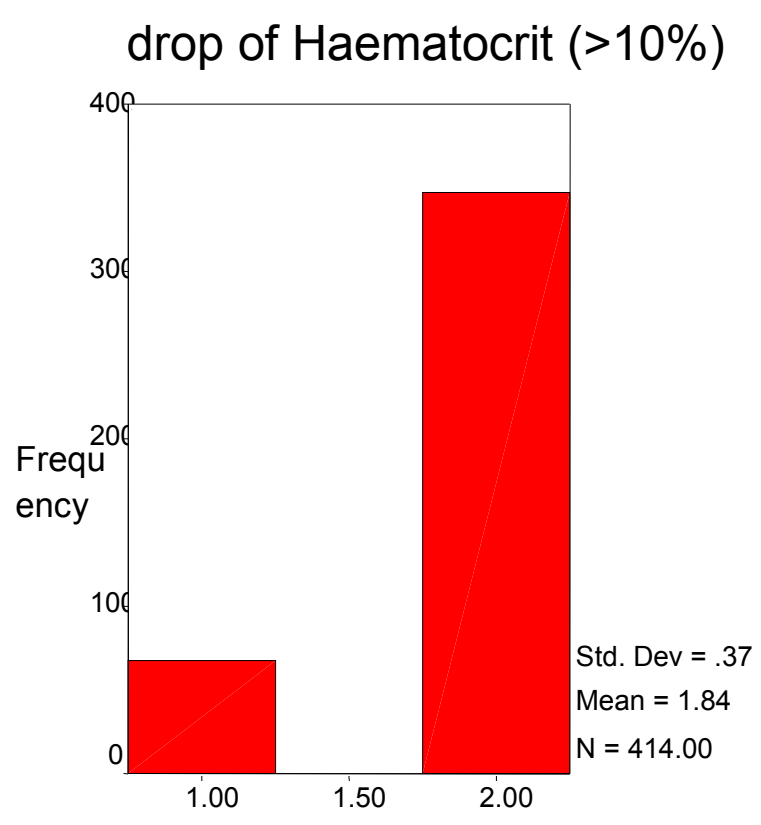

Drop of Haematocrit (>10\%)

Figure 1: Diagnosis of primary $\mathrm{PPH}$

\section{hut before dalinery}

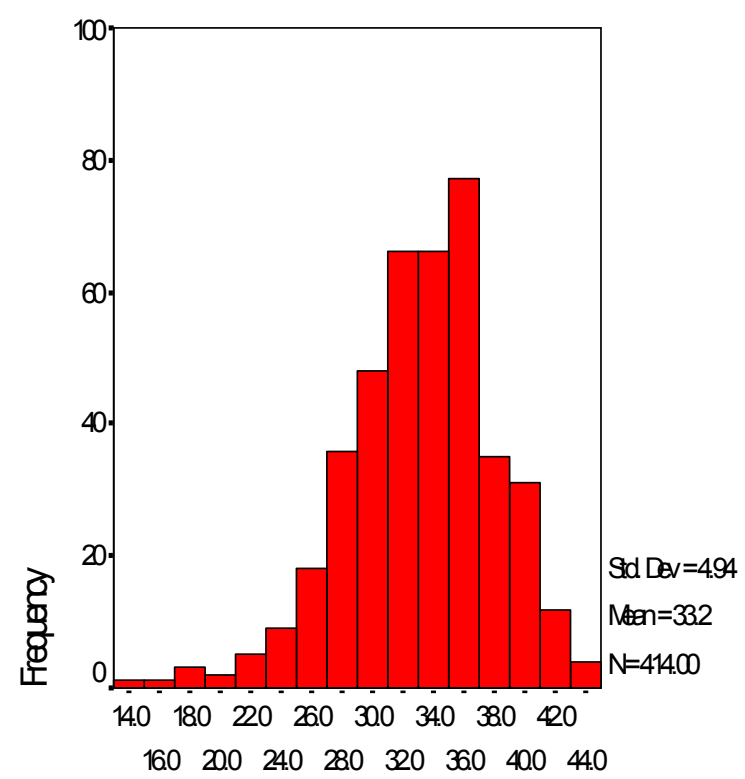

hat before delivery

\section{hot aterclinery}

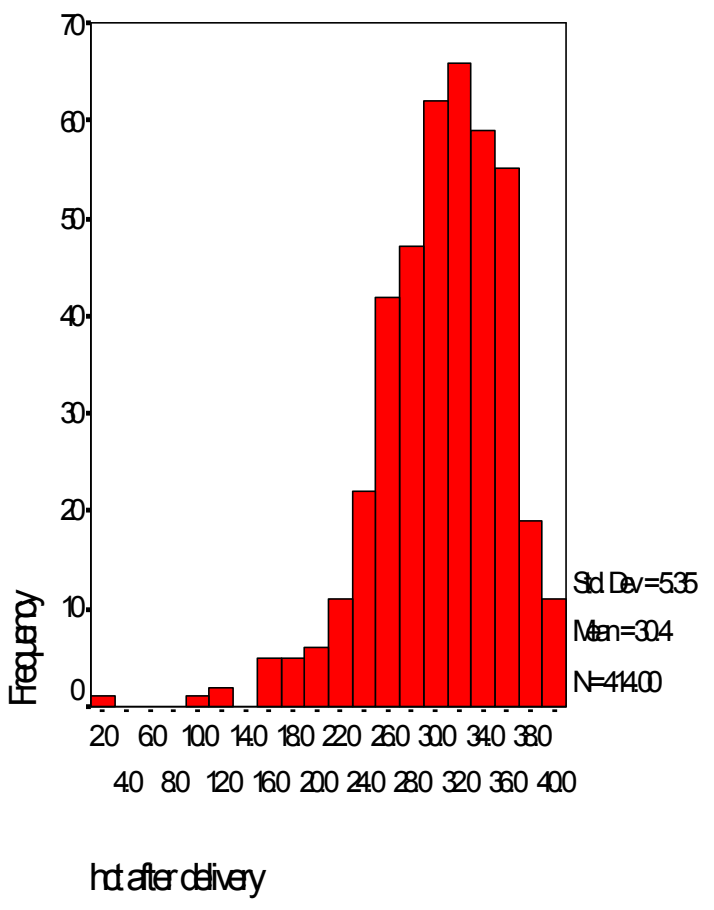

Figure 2: Change of haematocrit before and 12 hours after delivery 


\section{Discussion}

The study demonstrate a significant gap in diagnosis of primary PPH using visual estimation of blood loss during third stage of labour compared to the laboratory diagnosis. The average blood loss identified in the study (164.9) demonstrates an observer error especially when the non calibrated vessels are used to estimate blood loss. In our hospital the common vessel used in collecting blood loss after delivery is the kidney dish which is non-calibrated. Studies elsewhere using calibrated method demonstrated that there is improved accuracy in VEBL following prolonged use of calibrated vessels (Toledo et al., 2007). Muhimbili being a tertiary and teaching for both medical and nursing students need to upgrade their methods of estimating blood loss after delivery by providing the calibrated vessel so as to increase the long term memory in accurately estimating blood loss following delivery. Accurate diagnosis and prompt intervention of primary PPH is the key solution for prevention of maternal death related to PPH in most centres (Anderson \& Etches, 2007)).

In poor resource countries like Tanzania, primary PPH is the leading cause of maternal mortality despite of several interventions put in place. A study done in Thailand (Prasertcharoensuk et al., 2000) identified that the incidence of PPH by VEBL was 5.6\% compared to actual measurement of blood loss where the incidence was $27.6 \%$ which is not far from our finding when change in haematocrit was used The use of a change in haematocrit of more than $10 \%$ as a method of diagnosis of $\mathrm{PPH}$ compared to VEBL at delivery is more sensitive in diagnosis of PPH as demonstrated by some studies (Hofmeyr et al., 2005; Okonofua, 2005; Parsons et al., 2006). However this being retrospective method is not helpful for the immediate management of the patients hence leading to undetected morbidities which always lead to patient's readmission (Cameron \& Robson, 2006).

VEBL using non calibrated vessels have been reported to have significant observational errors, which often lead to underestimation of blood loss (Prata et al., 2005; Schorn, 2010). This method suffer from a theoretical teaching that vaginal blood loss following normal delivery is 200-500ml without conducting actual simulation where trainee has to measure actual blood loss followed by visual estimation. This teaching is also misleading when anaemic patients and patients with preeclampsia and eclampsia who are having contracted intravascular blood volume bleed, minimal bleeding to them means a lot compared to health women in labour (Higgins, 2003). Therefore this definition needs to be reviewed due to the fact that quantification of blood loss has several limitations ranging from the patients factor to the service providers factor. Most service providers tend to underestimates blood loss between $30-50 \%$ when there is excessive blood loss (Duthie et al., 1991; Razvi et al., 1996; Patel et al., 2006; Sharon et al., 2008).

In comparing PPH due to VEBL and change in haematocrit, our findings observed the error to be higher than in other studies (Razvi et al., 1996; Patel et al., 2006; Sharon et al., 2008)) but lower compared findings from studies by Toledo et al. (2007) and Yoong et al. (2010). This calls for a need to conduct a simulation study among services providers working in labour ward on accurate estimation of blood loss using both calibrated and non calibrated vessel (Dildy et al., 2004; Bose et al., 2006; Patel et al., 2006). The steady decline in the haematocrit change with an increase in the average VEBL was similar to findings by Gharoro \& Enabudoso (2009) but different from what was reported by Al Kadri et al. (2011). 
Despite of the high predictive values of detecting primary PPH by change in haematocrit, the study did not look at some of the factors which may affect change in haematocrit hence leading to high proportion of patient with PPH. The duration of labour which in most cases if it is prolonged is associated with dehydration is an important factor which was beyond the scope of this study. A significant number of women were on augmentation. The amount of fluid received during augmentation or during induction which also can affect the change of haematocrit leading to wrong diagnosis of PPH was not investigated. Patients who had complication during delivery and received some intervention like intravenous fluids and blood transfusion was not investigated to see how much was related with the change of haematocrit.

In conclusion, VEBL using calibrated vessel will increase accuracy where conventional method using non calibrated method is used for diagnosis of PPH. Service providers working in labour wards need to be trained on how to estimate blood loss using simulated methods so as to increase their long term memory and accuracy in diagnosis of post-partum haemorrhage, hence provision of immediate intervention.

\section{Acknowledgements}

The authors would like to thank Prudencia Maira and Josephine Tibatinzibwe for their assistance in data collection. Sued Masoud and David Mwanga are thanked for their technical assistance. Our thanks also go to all patients who participated in the study. We are grateful for financial support from Muhimbili University of Health and Allied Sciences and the National Institute for Medical Research.

\section{Declaration of interest}

None.

\section{References}

Al Kadri, H.M.F., Al Anazi, B.K. \& Tamim, H.M. (2011) Visual estimation versus gravimetric measurement of postpartum blood loss: a prospective cohort study. Archives of Gynecology and Obstetrics 283, 1207-1213.

Anderson, J.M. \& Etches, D. (2007) Prevention and management of postpartum hemorrhage. American Family Physician 15, 875-882.

Bose, P., Megan F. \& Peterson-Brown, S. (2006) Improving the accuracy of estimated blood loss at obstetric haemorrhage using clinical reconstructions. BJOG 113, 919-24.

Cameron, M.J. \& Robson, S.C. (2006) Vital statistics: an overview. In: Lynch, C-B, Keith, L., Lalonde, A., Karoshi, M. (eds) A Textbook of Postpartum Haemorrhage. Dumfries: Sapiens Publishing, pp. 17-34.

Chong, Y.S. \& Su, L.L. (2006) Misoprostol for preventing PPH: some lessons learned. Lancet 368, 1216-1218. 
Dildy, G.A., Paine, A., George, N.C. \& Velasco, C. (2004) Estimating blood loss: can teaching significantly improve visual estimation? Obstetrics and Gynecology 104, 601-606.

Duthie, S.J., Van, D., Yung, G.L., Guang, D.Z., Chan, S.Y. \& Ma, H.K. (1991) Discrepancy between laboratory determination and visual estimation of blood loss during normal delivery. European Journal of Obstetrics \& Gynecology and Reproductive Biology 38, 119-24.

Gharoro, E.P. \& Enabudoso, E.J.( 2009) Relationship between visually estimated blood loss at delivery and postpartum change in haematocrit. Journal of Obstetrics and Gynaecology 29, 517-520.

Higgins, S. (2003) Obstetric haemorrhage. Emerg Med (Fremantle), 15, 227-31.

Hofmeyr, G.J., Gülmezoglu, A.M., Novikova, N., Linder, V., Ferreira, S., Piaggio, G. ( 2009) Misoprostol to prevent and treat postpartum haemorrhage: a systematic review and meta-analysis of maternal deaths and dose-related effects. Bulletin of the World Health Organization 87, 666-677.

Hofmeyr, G.J., Walraven, G., Gulmezoglu, A.M., Maholwana, B., Alfirevic, Z. \& Villar, J. (2005) Misoprostol to treat postpartum haemorrhage: a systematic review. BJOG 112, 547-553.

Okonofua, F. (2005) Misoprostol and women's health in Africa. African Journal of Reproductive Health 9, 7-9.

Parsons, S.M., Walley, R.L., Crane, J.M. \& Matthews, K., Hutchens, D. (2006) Oral misoprostol versus oxytocin in the management of the third stage of labour. Journal of Obstetrics and Gynaecology Canada 28, 20-26.

Patel, A., Goudar, S.S., Geller, S.E., Kodhany, B.S., Wagh, K.,_Edlavitch, S.A., Patted, S.S., Naik, V.A., Moss, N. \& Derman, R.J. (2006) Drape estimation vs. visual assessment for estimating postpartum haemorrhage. International Journal of Gynaecology and Obstetrics 93, 220-224.

Pembe, A.B., Wangwe, J.T. \& Massawe, S.N. (2012) Emergency peripartum hysterectomies at Muhimbili National Hospital, Tanzania: a review of cases from 2003 to 2007. Tanzania Journal of Health Research 14 (1).

Prasertcharoensuk, W., Swadpanich, U. \& Lumbiganon, P. (2000) Accuracy of the blood loss estimation in the third stage of labour. International Journal of Gynaecology and Obstetrics 71, 69-70.

Prata, N., Mbaruku, G., Campbell, M.,Potts, M. \& Vahidnia, F. (2005) Controlling postpartum haemorrhage after home births in Tanzania. International Journal of Gynecology and Obstetetrics 90, 51-55.

Rath, W.H. (2011) Postpartum hemorrhage - update on problems of definitions. Acta Obstetrica et Gynecological Scandinavica 90, 421-428.

Razvi, K., Chua, S., Arulkumaran, S. \& Ratnam, S.S. (1996) A comparison between visual estimation and laboratory determination of blood loss during the third stage of labour. Australia and New Zealand Journal of Obstetrics and Gynaecology 36, 152-154.

Schorn, M.W. (2010) Measurement of blood loss: review of the literature. Journal of Midwifery and Women's Health, 55, 20-7.

Sharon, M., Gad B., Joseph B. L., Amitai Z. \& Ariel, M. (2008) Improved accuracy of postpartum blood loss estimation as assessed by simulation. Acta Obstetrics and Gynecology 87, 929934. 
Toledo, P., McCarthy, R.J., Hewlett, B.J., Fitzgerald, P.C. \&Wong, C.A. (2007) The accuracy of blood loss estimation after simulated vaginal delivery. Anesth Analg, 105(6), 1736-1740.

Yoong,W., Karavolos, S., Damodaram, M., Madgwick, K., Milestone, N., Al-Habib, A., Fakokunde, A. \& Okolo, S. (2010) Observer accuracy and reproducibility of visual estimation of blood loss in obstetrics: how accurate and consistent are health-care professionals? Archives of Gynecology and Obstetrics 281, 207-213. 